\title{
Fracture toughness and fracture mechanisms of polybutylene-terephthalate/polycarbonate/ impact-modifier blends
}

\author{
Part III Fracture toughness and mechanisms of PBT/PC \\ blends without impact modifiers
}

\author{
JINGSHEN WU, YIU-WING MAI \\ Centre for Advanced Materials Technology, Department of Mechanical and Mechatronic \\ Engineering, University of Sydney, Sydney, NSW 2006, Australia \\ ALBERT F. YEE \\ Department of Materials Science and Engineering, University of Michigan, Ann Arbor, \\ MI 48109, USA
}

\begin{abstract}
A series of polybutylene-terephthalate/polycarbonate (PBT/PC) blends with different compositions were prepared using a twin-screw extruder. The morphologies of the blends were revealed by scanning electron microscopy (SEM) and transmission electron microscopy (TEM). It was found that a 50/50 PBT/PC blend possessed a bicontinuous structure and the other blends had a dispersed phase of either PBT or PC depending on which was the minor component. A relatively strong interface was observed in the blends with $20 \%, 40 \%$ and $50 \%$ PBT; but poor interfacial adhesion was found in the blends with $60 \%$ and $80 \%$ PBT. The strength of the interfacial boundary was believed to depend on the composition and blending conditions of the individual blend. Fracture experiments showed that the sharp-notch fracture toughness of PC could be significantly increased by mixing with up to $50 \%$ PBT without losing its modulus and yield stress. The toughening mechanisms involved in the fracture processes of the blends were studied using both SEM and TEM together with single-edgedouble-notched-bend (SEDNB) specimens. It was found that in the toughened blends the growing crazes initiated by the triaxial stress in front of the crack tip were stabilized by the PC domains. The debonding-cavitation mechanism occurred at the PBT/PC interface, which relieved the plane-strain constraint and promoted shear deformation in both PBT and PC. This plastic deformation absorbed a tremendous amount of energy. Crack-interface bridging by the PC domains was clearly verified by the TEM study. Thus, the PC domains not only stabilized the growing crazes they also bridged crack surfaces after the crack has passed by. This effect definitely caused a large plastic-damage zone and hence a high crack resistance. Poor crack resistances of the blends rich in PBT was caused by the poor interfacial adhesion between PBT and PC. In these polymer blends, the growing crazes easily developed into cracks, which subsequently passed through the weak interface of PBT/PC and finally produced fast unstable fracture.
\end{abstract}

\section{Introduction}

Rigid-rigid polymer blends have received considerable attention since the early 1980 s because of their good balance between mechanical properties, fracture toughness and other important properties, such as chemical resistance and high temperature tolerance. One of the most important types of rigid-rigid polymer blends, which has been widely used in the automobile industry, consists of a series of compositions of polybutylene-terephthalate/polycarbonate impact-modifier (PBT/PC/IM). It is wellknown that polycarbonate is very tough, but it is also highly notch sensitive. The excellent toughness of PC originates from its low shear-yielding stress relative to its crazing resistance. Hence, the high toughness is not retained when the specimen is thick and the notch is sharp, since the shear deformation is suppressed by the plane-strain constraint under this particular condition [1]. Moreover, the solvent resistance of $\mathrm{PC}$ is poor, which limits its application in some areas. PBT is a semicrystalline polymer with remarkable chemical resistance and a wide processing window. In general, it is believed that the PBT/PC/IM blend inherits its toughness from $\mathrm{PC}$ and its chemical resistance from PBT, its superior high toughness at low temperatures is attributed to the impact modifier added. 
In Parts I and II of the present series $[2,3]$ the fracture behaviour of a commercial grade PBT/ $\mathrm{PC} / \mathrm{IM}$ blend was characterized under different testing conditions by fracture mechanics. The toughening mechanisms were studied via scanning electron microscopy (SEM) and transmission electron microscopy (TEM). Although an overall picture of the toughening mechanisms of the PBT/PC/IM blend is clearly described, the individual importance of the PBT, the PC and the impact modifier is still not certain and many important questions remain unanswered. For example, how does the PC phase impart toughness to the blend; what is the actual role of the PBT/PC interface during fracture; where should the optimal location for the impact modifier be: in the PBT domain or the PC domain? What is the most suitable domain size for the blend and is a bicontinuous structure superior to a matrix-particle structure. In the present study, a series of PBT/PC blends without the impact modifier were made in the laboratory using a twin-screw extruder. The mechanical properties and fracture toughnesses of the blends were investigated. The morphologies of the blends and the fracture mechanisms were studied using SEM and TEM together with the single-edge-double-notched-bend (SEDNB) technique. The effects of composition, blending condition, interface strength and domain size are discussed.

\section{Experimental work}

The polybutylene terephthalate (PBT) and polycarbonate $(\mathrm{PC})$ used in preparing the blends were supplied by G. E. Plastics and the Dow Chemical Company, respectively. The details of the resins and the composition of the blends are given in Table I. Prior to blending, the PBT and PC pellets were dried at $120^{\circ} \mathrm{C}$ for $5 \mathrm{~h}$ in an air-circulating oven. The dried pellets were first mixed manually and then blended in a twin-screw extruder (Werner and Pfleiderer ZSK-30, $L / D=29 / 1$, where $L$ is the total barrel length and $D$ is the barrel bore diameter) at predetermined temperatures and screw speeds, as shown in Table I. The single strand extrudate was pulled through a water bath and pelletized using a 2 inch pelletizer (Killion Extruders, Inc.). The pellets of the blends made were then dried at $120^{\circ} \mathrm{C}$ for $5 \mathrm{~h}$ and subsequently injection moulded into dog-bone bars for tensile tests (ASTM D-638) and rectangular bars for single-edge-notched-bend (SENB) tests.

The morphology of the blends was investigated using both SEM and TEM. The fracture surface used for the SEM study was perpendicular to the mouldfilling direction (MFD) of the injection-moulding bars and it was coated with gold before being studied in a Hitachi S-800 scanning electron microscope. Ultrathin sections for TEM analysis were cut using a Reichart Ultracut $\mathrm{E}$ ultramicrotome and the plane of the thin sections was also perpendicular to the MFD. After cutting, the thin sections were then mounted on copper grids and, for improved contrast, the specimens were exposed to an $\mathrm{RuO}_{4}$ vapour for $10 \mathrm{~min}$. The TEM study was carried out in a Phillips E-420.

The tensile properties of the blends were determined according to ASTM D-638 using an Instron 4502 machine with a computerized data-acquisition system at ambient temperature $\left(22-25^{\circ} \mathrm{C}\right)$ and the crosshead speed was $10 \mathrm{~mm} \mathrm{~min}^{-1}$. Fracture-toughness values were obtained at the same temperature using the specific-essential-fracture-work concept described in Part I [2]. The geometry of the specimens for the specific-essential-fracture-work tests were $6.4 \times 12.75$ $\times 63.85 \mathrm{~mm}^{3}$ and $3.2 \times 12.75 \times 63.85 \mathrm{~mm}^{3}$, respectively. A sharp notch was made on one side of each specimen using the technique given in [2]. Details of the fracture-toughness measurement can be found in [2].

Toughening mechanisms were studied using the (SEDNB) technique together with TEM and SEM. TEM specimens were also stained with $\mathrm{RuO}_{4}$ vapour. The experimental details of the SEDNB technique can be found in Part 2[3] and elsewhere $[4,5]$.

\section{Results and discussion}

\subsection{Morphology study}

A morphology study was carried out using both SEM and TEM samples. The observation plane of the specimens was prepared in the direction perpendicular to the MFD; the SEM and TEM photographs shown in Figs 1 to 5 were taken from the core area of the injection-moulded bars.

Fig. 1a is a TEM micrograph of a PBT/PC (20/80) blend. The black continuous phase is $\mathrm{PC}$ which was stained by $\mathrm{RuO}_{4}$, and white dispersing particles are

TABLE I Processing conditions and compostions of the PBT/PC blends

\begin{tabular}{|c|c|c|c|c|c|c|c|c|}
\hline \multirow{2}{*}{$\begin{array}{l}\text { Material } \\
\text { Code }\end{array}$} & \multirow{2}{*}{$\begin{array}{l}\text { PBT } \\
(\%)\end{array}$} & \multirow{2}{*}{$\begin{array}{l}\mathrm{PC} \\
(\%)\end{array}$} & \multicolumn{5}{|c|}{ Processing temperatures $\left({ }^{\circ} \mathrm{C}\right)$} & \multirow{2}{*}{$\begin{array}{l}\text { Screw speed } \\
(\mathrm{rpm})\end{array}$} \\
\hline & & & Zone 1 & Zone 2 & Zone 3 & Zone 4 & DIE & \\
\hline $\mathrm{PBT} / \mathrm{PC}(0 / 100)$ & 0 & 100 & & & & & & \\
\hline PBT/PC $(20 / 80)$ & 20 & 80 & 240 & 260 & 265 & 260 & 250 & $19-22$ \\
\hline $\mathrm{PBT} / \mathrm{PC}(40 / 60)$ & 40 & 60 & 240 & 260 & 265 & 260 & 250 & $19-22$ \\
\hline $\mathrm{PBT} / \mathrm{PC}(50 / 50)$ & 50 & 50 & 240 & 260 & 260 & 250 & 240 & 19. 22 \\
\hline $\mathrm{PBT} / \mathrm{PC}(60 / 40)$ & 60 & 40 & 230 & 250 & 260 & 240 & 230 & $19-22$ \\
\hline $\mathrm{PBT} / \mathrm{PC}(80 / 40)$ & 80 & 20. & 220 & 240 & 260 & 240 & 220 & $19-22$ \\
\hline $\mathrm{PBT} / \mathrm{PC}(100 / 0)$ & 100 & 0 & & & & & & \\
\hline
\end{tabular}



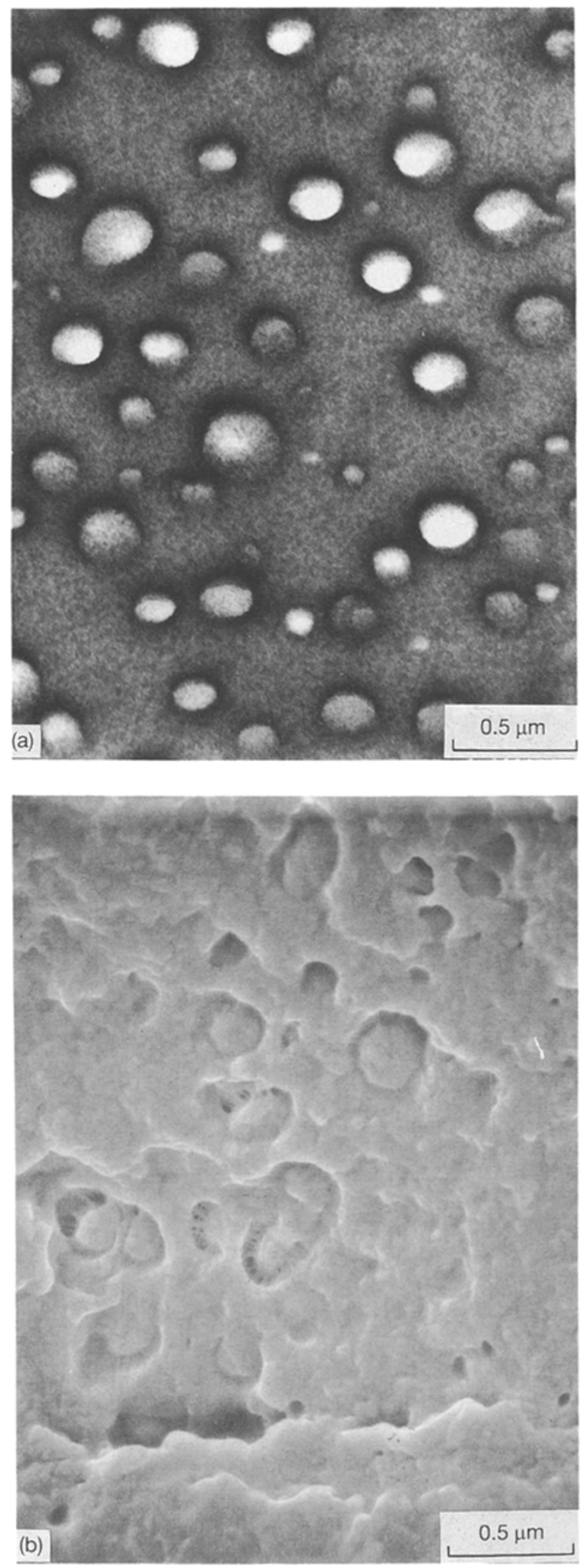

Figure 1 The morphology of a PBT/PC (20/80) blend by : (a) TEM, and (b) SEM. The black phase in the TEM micrograph represents $\mathrm{PC}$ stained by $\mathrm{RuO}_{4}$.

PBT, which are quite randomly and uniformly distributed in the PC matrix. The particle size was about $0.1-0.2 \mu \mathrm{m}$. The interfacial boundary between PC and PBT can be found in the SEM micrograph taken from a fracture surface of the blend, Fig. 1b. In Fig. 1,
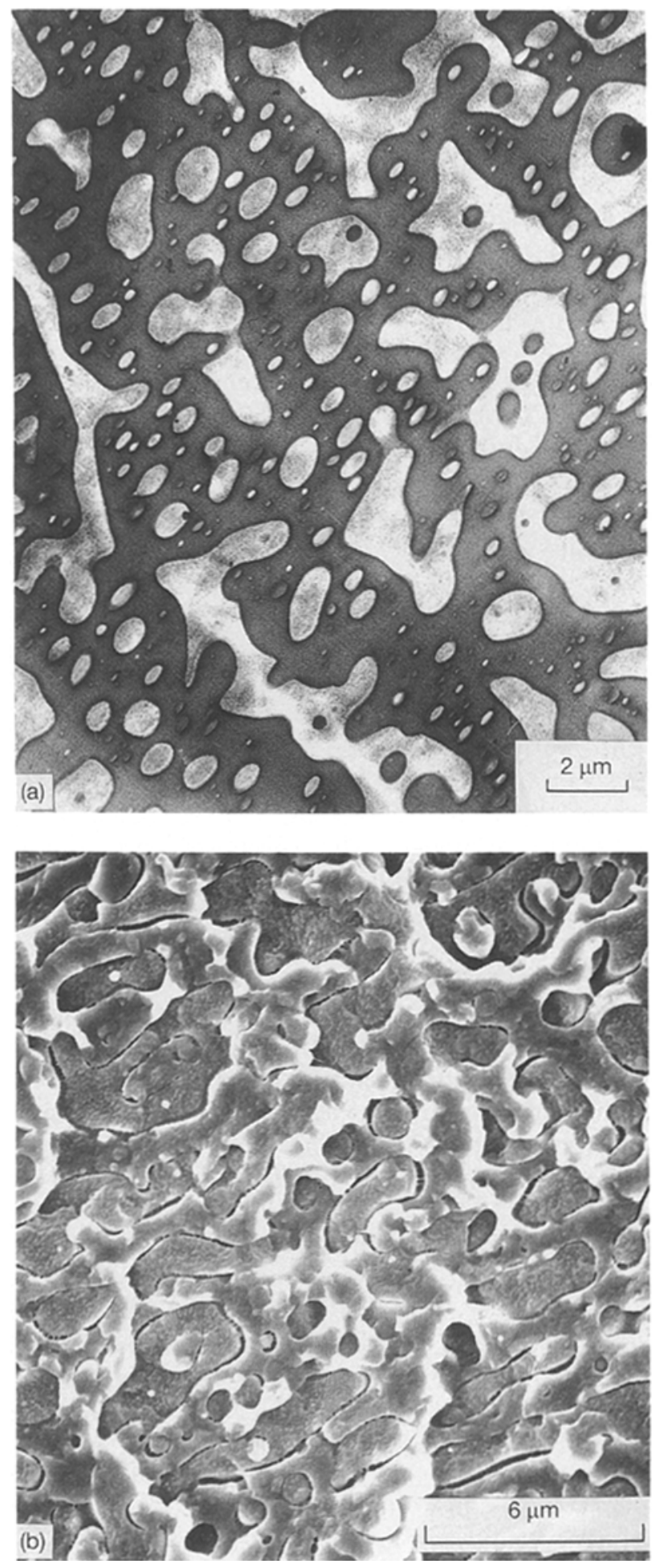

Figure 2 The morphology of a PBT/PC (49/60) blend by: (a) TEM, and (b) SEM. The black phase in the TEM micrograph represents PC stained by $\mathrm{RuO}_{4}$

debonding is seen and there are fibrils at the interface between the PBT particles and the PC matrix, giving a qualitative indication that the bond strength of the PBT/PC interface is good. PBT/PC is not a miscible system [6-9]; this means that the PBT/PC mixture will be in an unstable situation in processing if the polymers are stabilized against exchange reactions. In the present study, the blending of the $\mathrm{PBT} / \mathrm{PC}$ was conducted without a transesterification inhibitor, also, under the processing conditions shown 

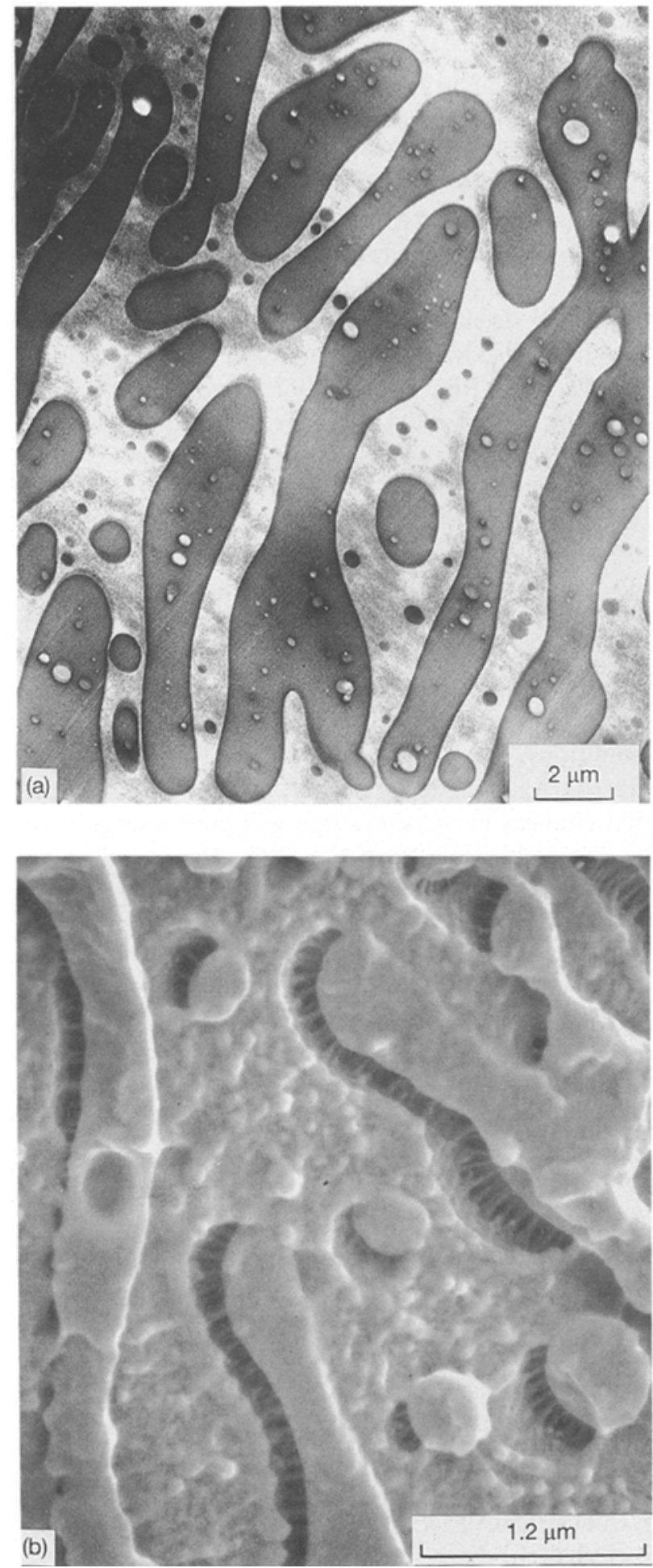

Figure 3 The morphology of a PBT/PC (50/50) blend: (a) TEM, and (b) SEM. The black phase in the TEM micrograph represents PC stained by $\mathrm{RuO}_{4}$. Strong interfacial adhesion can be seen in the SEM photograph.

in Table I, the dwelling time of the PBT/PC components during extrusion was about $4-5 \mathrm{~min}$, which is somehow sufficient for a certain extent of exchange reactions between PBT and PC to occur [7, 10-12]. Thus, it is a reasonable inference that the strong interface found in this blend might be the result of a copolymer generated by the transesterification between PBT and PC during extrusion $[6,7,10,11]$. Figs 2 and 3 show the microstructures of the blends containing $40 \%$ PBT $-60 \% \mathrm{PC}$ and $50 \% \mathrm{PBT}-50 \% \mathrm{PC}$, respectively.
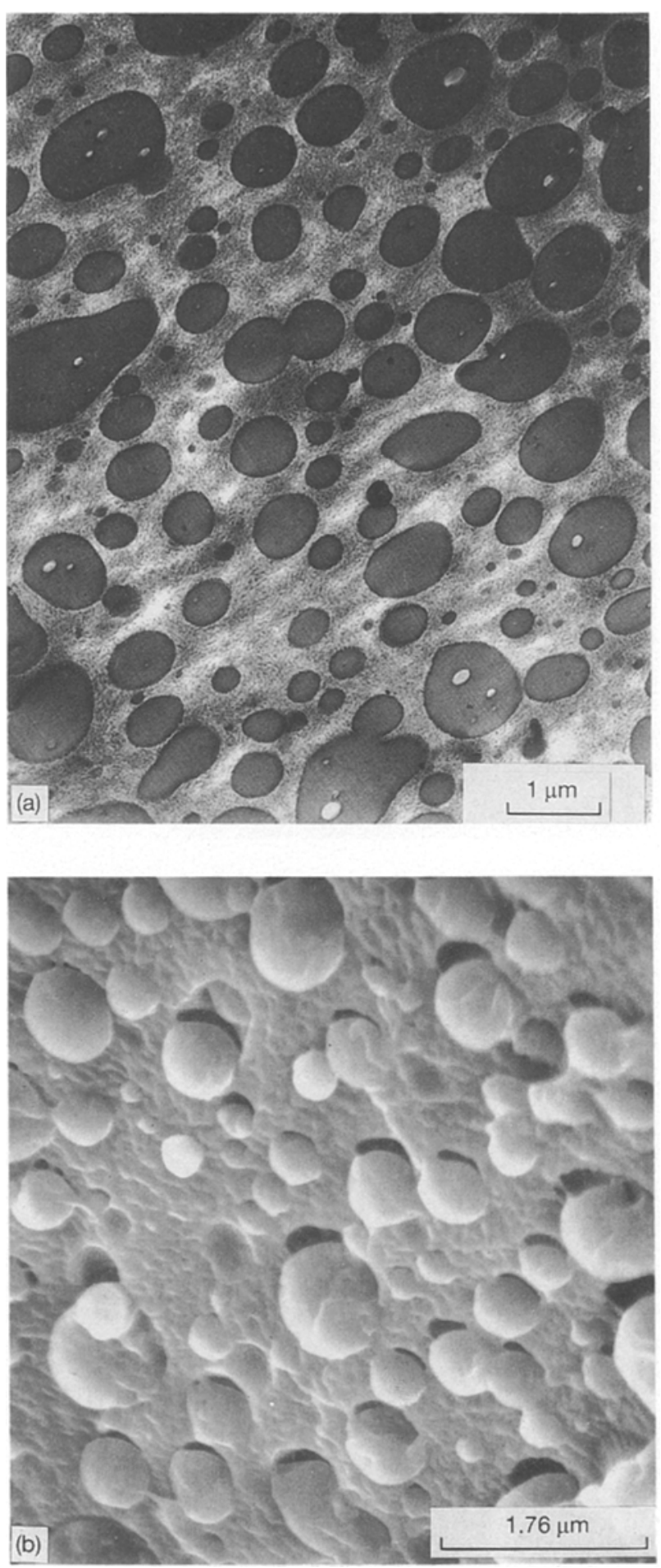

Figure 4 The morphology of a PBT/PC $(60 / 40)$ blend by: (a) TEM, and (b) SEM. The black particles in the TEM picture represent PC stained by $\mathrm{RuO}_{4}$. The interfacial adhesion between PBT and PC has decreased compared to that in the $(50 / 50)$ blend of Fig. 3, as shown by the SEM micrograph.

As expected, although the PBT phase becomes larger and it is no longer spherical in the PBT/PC $(40 / 60)$ blend (Fig. 2), PC is still the continuous phase. The PBT domain has a wide size range, from a couple to several tens of micrometres. A bicontinuous structure is observed in Fig. $3 \mathrm{a}$ for the $50 \% \mathrm{PBT}-50 \% \mathrm{PC}$ blend. The interface bond strength in these two blends is qualitatively similar to that found in the $20 \%$ PBT- $80 \%$ PC blend. As shown in Fig. 3b, the debonded $\mathrm{PBT}$ and $\mathrm{PC}$ domains were connected by many 

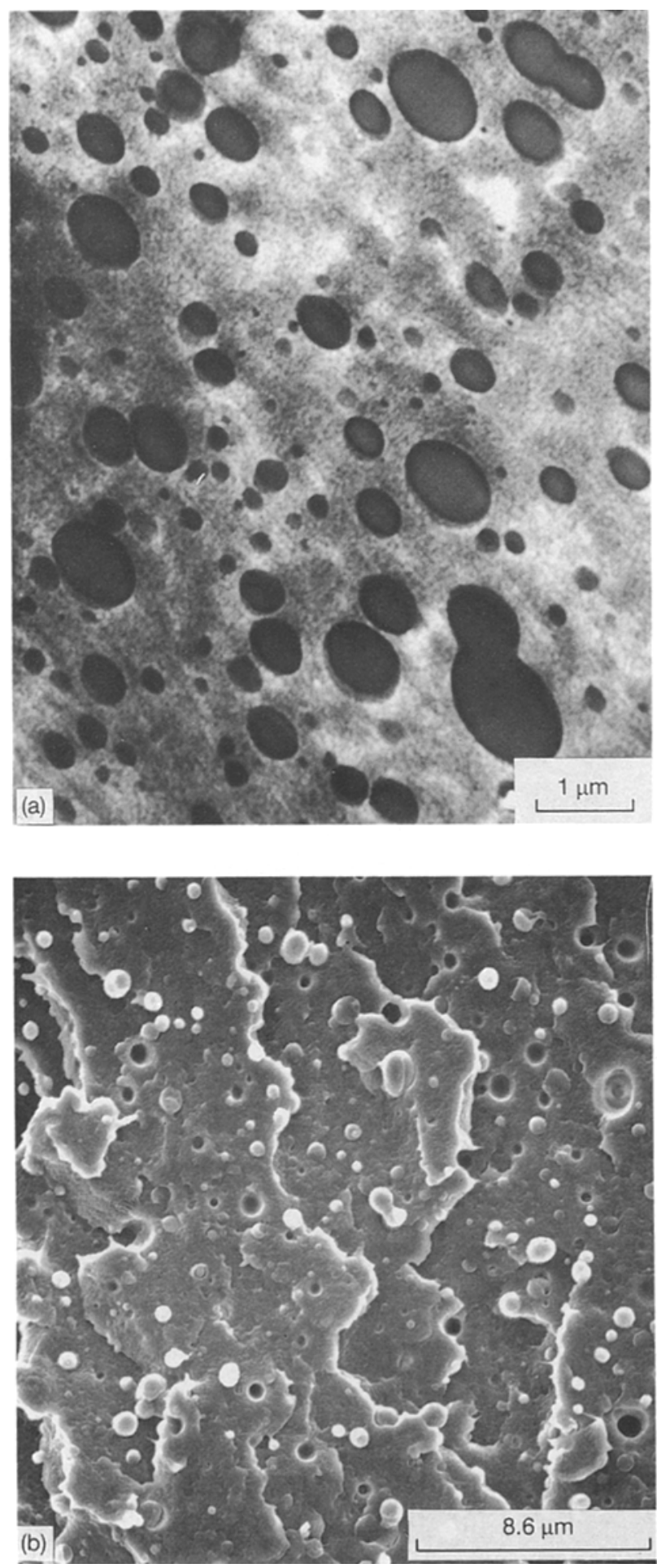

Figure 5 The morphology of a PBT/PC (80/20) blend: (a) TEM, and (b) SEM. The black particles in the TEM picture represent $\mathrm{PC}$ stained by $\mathrm{RuO}_{4}$. Poor adhesion between PBT and $\mathrm{PC}$ is evident in (b).

stretched polymer fibrils, confirming the probable existence of a relatively strong interface between PBT and PC. As in the case of the $20 \%$ PBT $-80 \%$ PC blend, the connecting polymer fibrils are suspected to be PBT/PC copolymers generated by PBT/PC transesterification during extrusion, although there is no direct evidence of the copolymer existence available in the present study. Figs 4 and 5 show the morphology of blends with an even greater proportion of PBT, $60 \%$ PBT $-40 \%$ PC and $80 \%$ PBT $-20 \%$ PC. The significant changes found in these two blends, compared with the blends discussed previously, are that the PC becomes the dispersed phase and the interfacial adhesion is poor. As shown in Figs $4 \mathrm{~b}$ and $5 \mathrm{~b}$, there is no bridging polymer fibril visible, instead many hemispherical holes with a smooth surface are found. Obviously, these hemispherical holes were formed after the PC domains were pulled off during fracture, and the smooth surface of the holes indicates that the bonding between PBT and PC was relatively poor. This poor interfacial strength can plausibly be attributed to the low melt viscosity found in the PBT/PC blending process when PBT is the major component.

As shown in Table I, the PC resin used in this study has a much higher melt viscosity $\left(3 \times 10^{3} \mathrm{~N} \mathrm{~m}^{-2}\right)$ than that of the PBT resin $\left(7.5 \times 10^{2} \mathrm{~N} \mathrm{~m}^{-2}\right)$ at the processing temperature range, $220-265^{\circ} \mathrm{C}$. Under the set of extrusion conditions used, the shear rates are normally below about $1000 \mathrm{~s}^{-1}$ and the melt viscosity of the $\mathrm{PC}$ resin is essentially Newtonian. This means that the melt viscosity of the $\mathrm{PC}$ is more sensitive to the processing temperature than to the shear rate. However, when the PBT is processed under the same conditions, the melt viscosity of the PBT is low and, moreover, it is basically non-Newtonian, which means that changes in the shear rate will have a large effect on the melt viscosity of the PBT. Hence, when the blends with PC as the major component were processed in an extruder at the temperature range above, the melt viscosity should be high; as a result, the frictional heat generated during mixing is expected to produce a localized high-temperature zone. Therefore, the actual temperature of the molten PBT/PC may be higher than the extruder barrel temperature. If it is assumed that similar PBT/PC transesterification does exist during extrusion blending, as has been suggested by Devaux et al. $[7,8,10,11]$, then the localized high temperature will accelerate the transesterification between PBT and $\mathrm{PC}$ and produce more $\mathrm{PBT} / \mathrm{PC}$ copolymers to increase the interfacial strength. Furthermore, the higher shear stress required in highviscosity processing introduces severe polymer chain scission and also gives the material system more energy to promote the exchange reaction between PBT and PC. By contrast, the PBT-rich blends generally possess a low melt viscosity when they are mixed in an extruder. Thus, frictional heat in this case is low and, also, a high shear stress cannot be built up in the material system because the higher the shear stress is the lower is the melt viscosity. The temperature of the molten PBT/PC system is therefore expected to be lower than the preferred reaction temperature of PBT/PC transesterification to occur. Hence, little copolymer, if any, will be created during processing.

\subsection{Mechanical properties and toughening mechanisms}

The yield stress and Young's modulus obtained in the tensile tests at ambient temperature $\left(22-25^{\circ} \mathrm{C}\right)$ are plotted against the percentage of PBT in Fig. 6. In the absence of significant chemical degradation, the modulus of the blend should not change with the percentage of PBT because the two parent polymers 


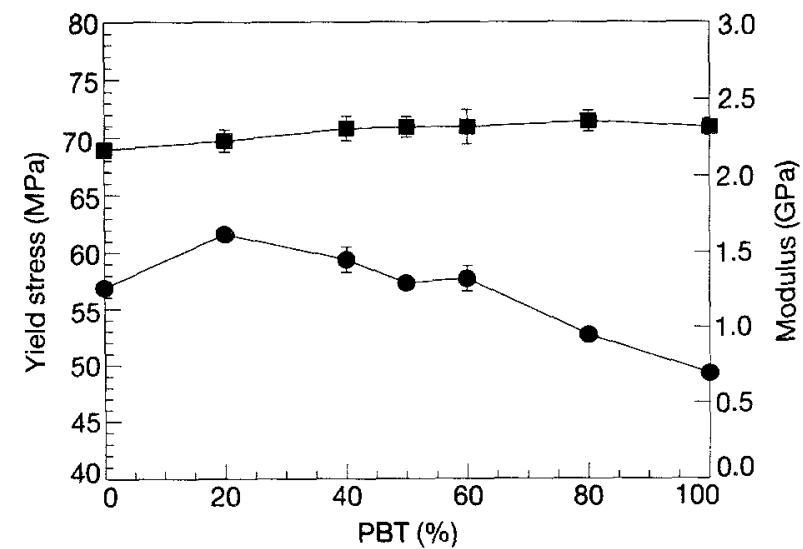

Figure 6 The mechanical properties of PBT/PC blends showing the variation of ( $\square$ ) the Young's modulus and $(O)$ the yield strength with the percentage of PBT. Tests were carried out at ambient temperature $\left(22-25^{\circ} \mathrm{C}\right)$ with a crosshead speed of $10 \mathrm{~mm} \mathrm{~min}^{-1}$.

have almost identical modulus values. The results are consistent with this expectation. A slight increase in the yield stress was found when $20 \%$ PBT was added. But further increases in the amount of PBT caused the yield stress of the blends to drop down continuously to the yield stress of pure PBT.

The variation of fracture toughness (in terms of the specific essential fracture work, $w_{\mathrm{e}}$ ) with the PBT content is shown in Fig. 7. In this sharp-notch toughness test, $\mathrm{PC}$ appears to be very brittle and sensitive to the specimen thickness. For the $3.2 \mathrm{~mm}$ thick specimens the toughness of PC was about $4.1 \mathrm{~kJ} \mathrm{~m}^{-2}$, but when the thickness of the samples was $6.4 \mathrm{~mm}$ it was only $1.7 \mathrm{~kJ} \mathrm{~m}^{-2}$. However, the addition of an appropriate amount of PBT increased the fracture toughness drastically. For instance, in the fracture tests carried out on $6.4 \mathrm{~mm}$ thick specimens, adding $20 \%$ PBT to $80 \% \mathrm{PC}$ improved the fracture toughness by a factor of four. The specific essential fracture work, $w_{\mathrm{e}}$, in this case was $7 \mathrm{~kJ} \mathrm{~m}^{-2}$. Even more substantial toughness enhancement was obtained by mixing $60 \% \mathrm{PC}$ with $40 \% \mathrm{PBT}$, the fracture toughness of this particular blend was about $11.2 \mathrm{~kJ} \mathrm{~m}^{-2}$, which is six times larger than for pure PC. After this point, further increases in the amount of PBT reduced the fracture toughness of the blends gradually to about $2.5 \mathrm{~kJ} \mathrm{~m}^{-2}$ for the $80 \%$ PBT $-20 \%$ PC blend. The variation of the fracture toughness with the PBT percentage obtained using $3.2 \mathrm{~mm}$ thick specimens had a similar trend to that described for the $6.4 \mathrm{~mm}$ thick specimens.

The toughening mechanisms related to the abovementioned phenomena are illustrated in Figs 8-13. Fig. 8 shows SEM micrographs of a fracture surface of pure PC. As expected, only a few river lines can be seen, and a large area of the fracture surface is relatively smooth and featureless. There is little significant energy-absorption-related deformation mechanism because the plane-strain constraint limits the shear deformation of PC,

Compared to the featureless fracture surface of a pure PC, the SEM micrographs of the crack-initiation area of a fracture surface of a PBT/PC (20/80) blend, Fig. 9, show much greater plastic deformation and cavitation. The fracture surface in this case is no

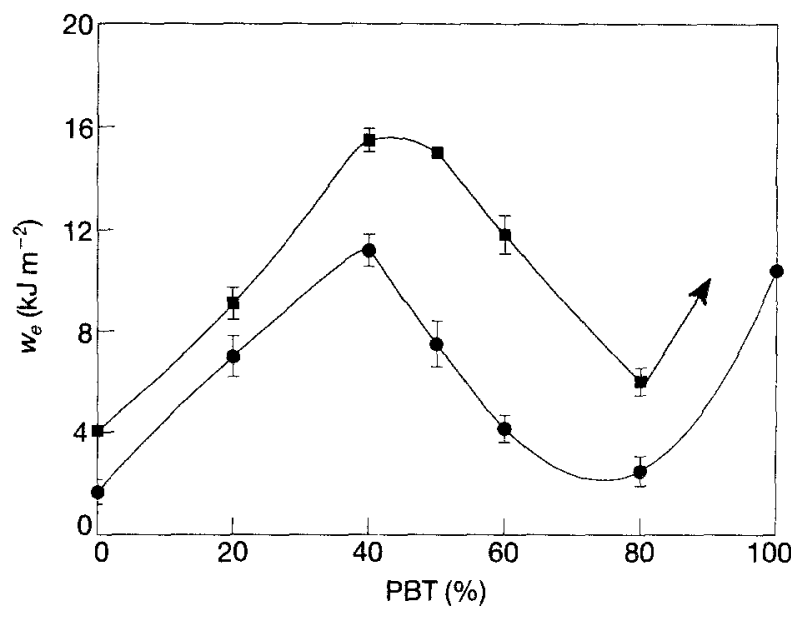

Figure 7 The variation of the specific essential fracture work, $w_{e}$ with the PBT percentage for two specimen thicknesses: (a) $B=3.2 \mathrm{~mm}$, and (O) $B=6.4 \mathrm{~mm}$. Tests were carried out at ambient temperature $\left(22-25^{\circ} \mathrm{C}\right)$ with a crosshead speed of $5 \mathrm{~mm} \mathrm{~min}^{-1}$.

longer smooth. Tiny voids formed via debonding are visible in Fig. 9a, and in the enlargement, Fig. 9b, a typical fibrillated structure caused by extensive plastic stretching and tearing can be clearly observed. Presumably, it is this plastic deformation which is responsible for the toughness enhancement shown in Fig. 7.

Fig. 10a and $\mathrm{b}$ illustrates the toughening mechanisms involved in the fracture processes of the PBT/PC (40/60) blend. The entire fracture surface shown in Fig. 10a consists of two distinct zones, namely, a cavitation zone in the middle core area and a shear zone in the outer skin area. The formation of the cavitation zone implies that there had been a triaxial stress field when the sample was loaded prior to the propagation of the initial crack. The close-up view of the cavitation zone in Fig. 10b reveals that widespread cavitation occurred at the boundary between the PBT and PC domains. Some very large voids were formed via the coalescence of neighbouring small holes. Evidently, the triaxial-stress state in the centre area was relieved by this cavitation and coalescence mechanism and general shear deformation followed. Very similar toughening mechanisms were also found in the fracture processes of the PBT/PC (50/50) blend, see Fig. 10c to e. However, when the micrographs are studied more closely, it can be noted that there are many fibre-like broken pieces standing in the holes and surrounded by highly deformed material, indicated by the arrows in Fig. 10d and e. Although the rod-like PBT and PC domains, as illustrated in Figs 2 and 3, were formed in extrusion and injectionmoulding cycles, prior to the fracture event, it seems that one of the two parent-polymer rod-like domains, either PBT or PC, had been stretched into fibrils during the crack-opening process and had broken after fracture of the surrounding material. In other words, crack bridging might have happened during crack propagation.

Since SEM cannot give a satisfactory answer to this hypothesis, a SEDNB specimen was employed with TEM for further analysis. The preparation of the 

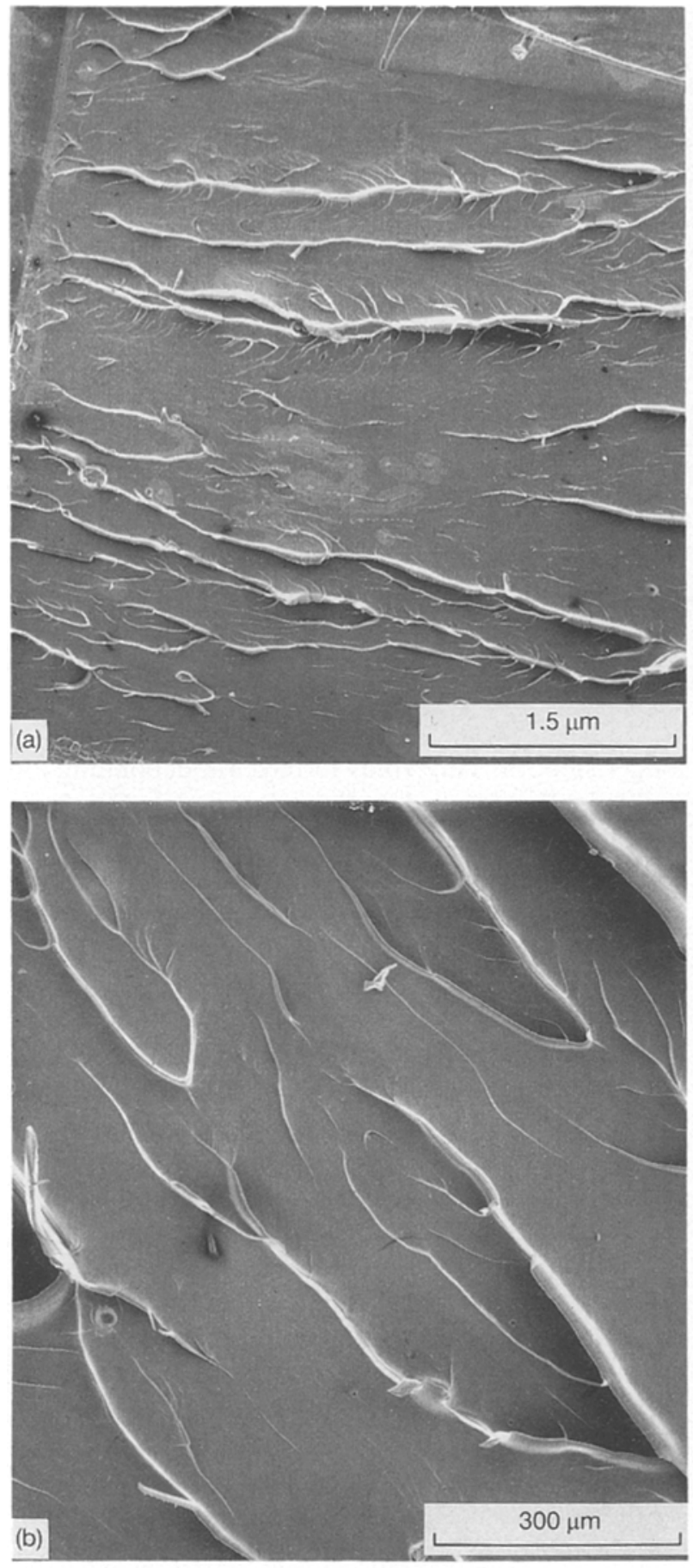

Figure 8 SEM micrographs of the fracture surface of a pure PC sample. The fracture surface is relatively smooth.

TEM specimens is sketched in Fig. 11. Fig. 12 shows TEM micrographs taken from a $\mathrm{RuO}_{4}$ stained ultrathin section containing an arrested crack tip. From these micrograph the following points can be made. (i) Crack bridging did occur during the fracture of the blends. (ii) It is the PC domains that were stretched into fibres and they bridge the crack faces. As shown in Fig. 12a and b, the black PC domains link up the crack faces, and shear yielding is found in these bridging particles. It is also clear from these micrographs that the PBT domains were already broken at this stage. (iii) Crazes found in front of a propagating crack were stabilized by the bridging PC domains before PBT/PC debonding could occur. Fig. $12 \mathrm{c}$ is a micrograph taken from the area right in front of a propaga-
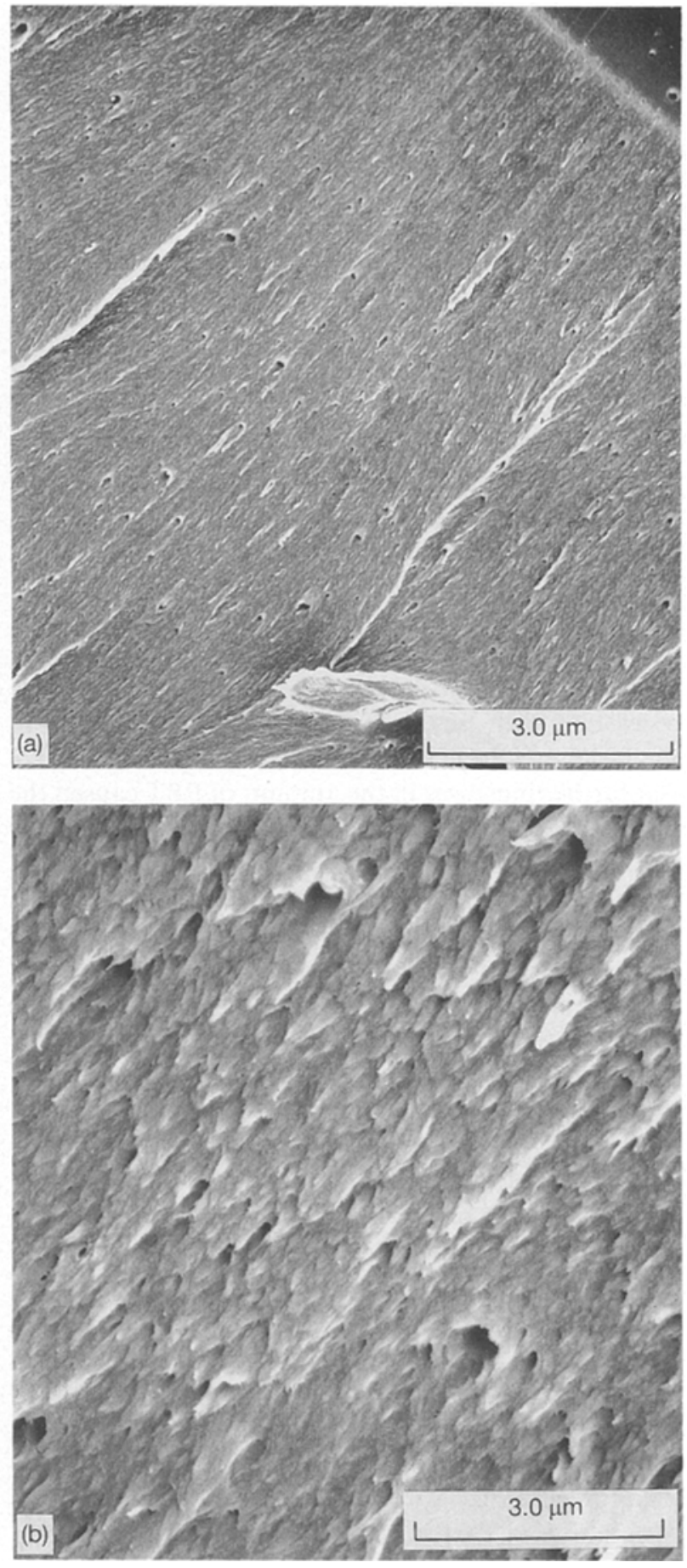

Figure 9 SEM micrographs of a PBT/PC (20/80) blend: (a) the fracture surface, and (b) the plastic deformation in the crackinitiation area (which is responsible for the toughness enhancement).

ting crack tip. Several crazes were initiated and they radiated from the crack tip and were finally stabilized by the black PC domains. However, no debonding is visible.

From the discussion above, it may be concluded that the substantial increase in toughness observed in the PBT/PC (40/60) and PBT/PC (50/50) blends has contributions from several toughening mechanisms. Among these are debonding-cavitation, massive shear deformation of both PBT and PC, and crack bridging. The toughening processes in these cases may be described as follows. It is known that when a cracked sample is subjected to loading a triaxial stress field will 
build up in front of the crack tip and if there is no triaxial-stress relief brittle failure will eventually occur. In the present study, the triaxial stress first induces craze initiation in the PBT phase, but these crazes are stabilized by the PC domains. Hence, the triaxial stress rises to a higher level and subsequently causes debonding and introduces localized voids at the PBT/PC phase boundary, as shown in Fig. 13a. The localized tiny voids will continue to expand under the increasing triaxial stress, until the polymer ligaments between the neighbouring voids become thin enough to enable shear deformation of the ligament to occur (as shown in Fig. 13b). At this stage, massive shear plastic deformation takes place in both PBT and PC and this absorbs a tremendous amount of energy. Further increases in the applied load finally cause failure of the PBT domains. However, the PC domains at this time are only stretched into fibres and continue to bridge the crack surfaces, Fig. 13c. Therefore, the bridging domains not only prevent crazes from growing into harmful cracks but they also bridge the crack faces and continuously transfer stress to the material in the vicinity of the crack faces after the main crack has passed by. All these toughening mechanisms definitely increase the damage-plastic-zone size and enhance the total fracture toughness.

Fig. 14 shows micrographs taken from the fracture surface of the PBT/PC (60/40) blend. The plastic
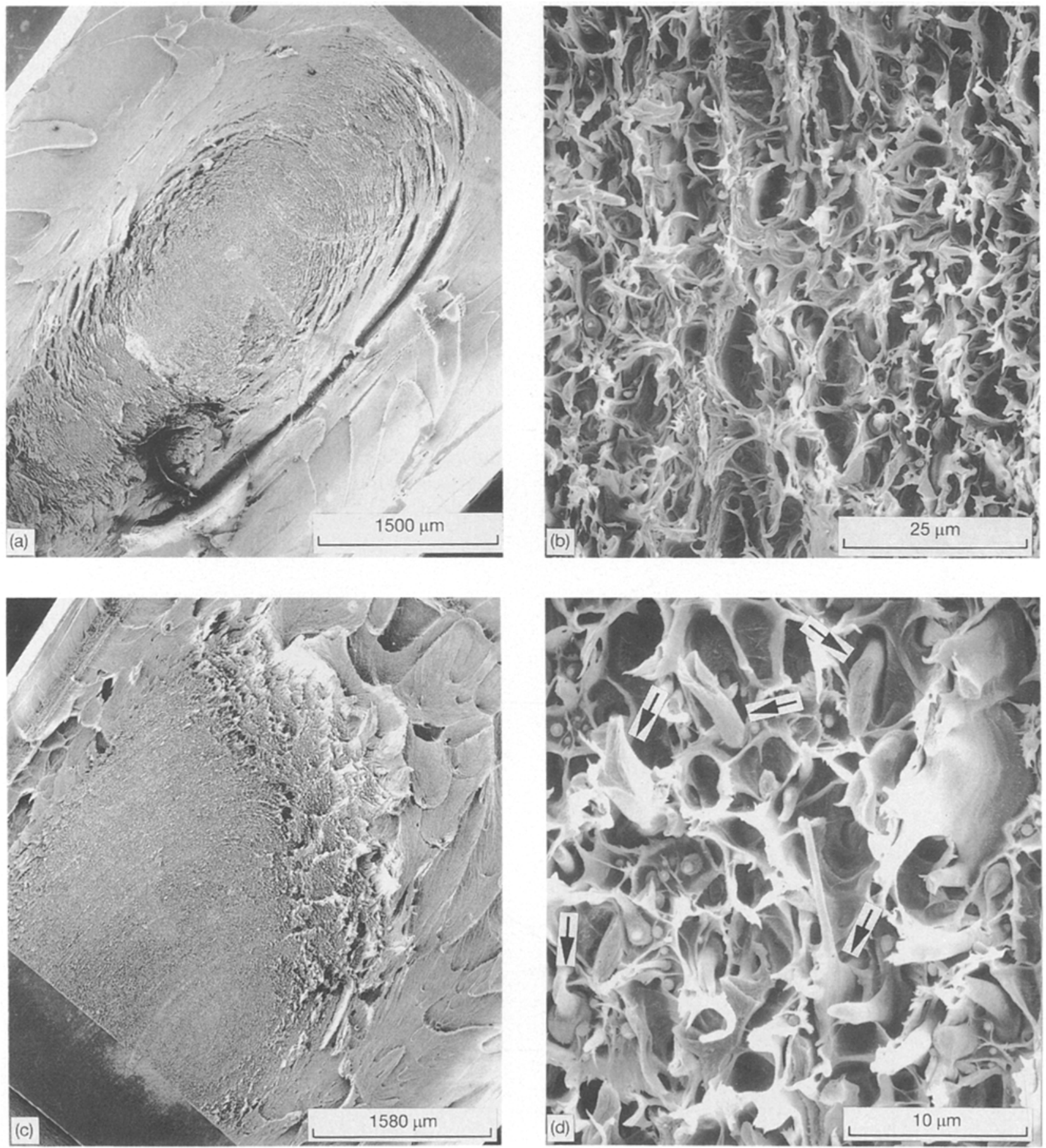

Figure 10 SEM micrographs taken from the fracture surfaces of the PBT/PC (40/60) and (50/50) blends. (a) and (c) show two distinct zones on the fracture surfaces of PBT/PC (40/60) and (50/50) blends, respectively. Extensive cavitation and plastic deformation can be observed in the crack initiation area in (b) to (e). Bridging domains were stretched into fibrils before breaking, as indicated by the arrows in (d) and (e). 


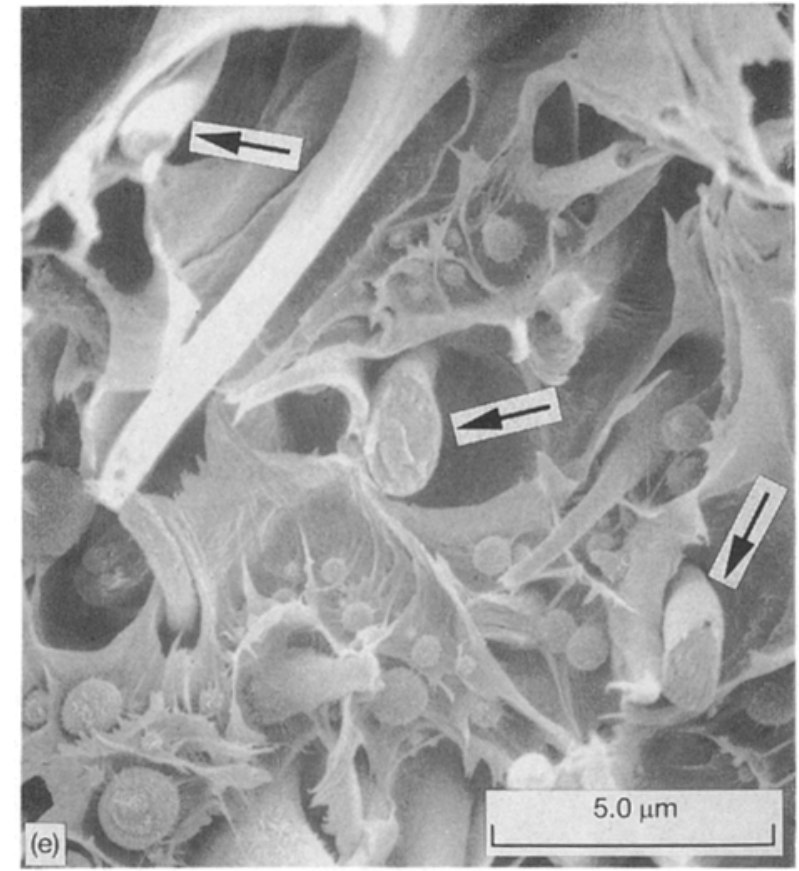

Figure 10 (Continued).

deformation shown in Fig. 14b was found only in a very small whitening area, indicated by the arrow in Fig. 14a The rest of the picture (Fig. 14a) shows a typical unstable fast fracture surface. A higher magnification micrograph from this unstable fracture area, Fig. 14c, reveals that the prevalent interfacial debonding between PBT and PC was due to the poor interfacial adhesion, as discussed in Section 3.1. Numerous hemispherical PC nodules were found on the fracture surface. They were formed by pull-out from the counterpart fracture surface. No evidence of crack bridging was visible in this particular blend. Therefore, it is suggested that when a cracked sample of this blend is subjected to loading the crazes in front of the crack tip, again initiated by the high triaxial-stress, quickly grow and become harmful cracks. These cracks will then pass through the interface of $\mathrm{PBT} / \mathrm{PC}$, which is the weakest path in this blend, and will eventually result in a fast unstable fracture and give the blends a low fracture toughness. These fracture processes are given schematically in Fig. 15.

The fracture behaviour of the PBT/PC (80/20) blend is very similar to that of the PBT/PC (60/40) blend. But the fracture surface of this blend is even smoother, Fig. 16a. The micrograph taken from the crack-initiation area, Fig. 16b, shows very limited plastic tearing with many debonded PC particles. In the fast unstable fracture area, Fig. $16 \mathrm{c}$, both debonded particles and hemispherical holes are clearly seen. The reasons responsible for the very low toughness found in this blend are the same as those discussed in the last paragraph.

To summarise the toughening mechanisms of all the $\mathrm{PBT} / \mathrm{PC}$ blends tested, it is suggested that the fracture processes in the PBT/PC blends occurred as follows. First, crazes formed in the PBT phase and at the crack tip. Secondly, if the interfacial adhesion between PBT and PC was good then the crazes were stabilized by the PC domains and prevented from growing into critical cracks; otherwise, the crazes developed into cracks and resulted in fast unstable fracture, as found in PBT/PC (60/40) and (80/20) blends. Thirdly, in the case of good interfacial adhesion, since the crazes are stabilized, the triaxial stress will rise to a higher level during loading until debonding occurs at the PBT/PC interface. Thus debonding-cavitation occurs and localized voids are formed and expanded until the
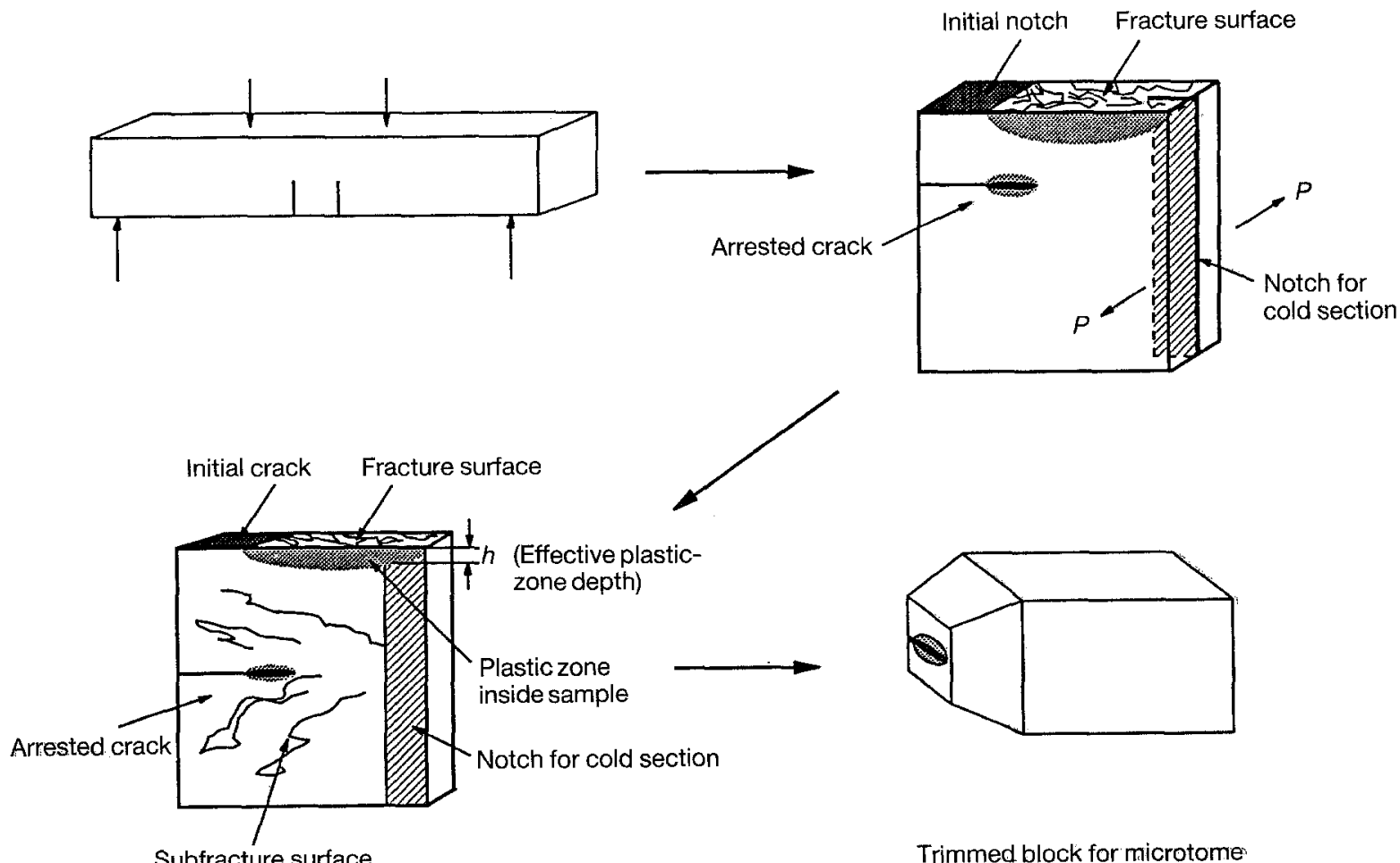

Subfracture surface

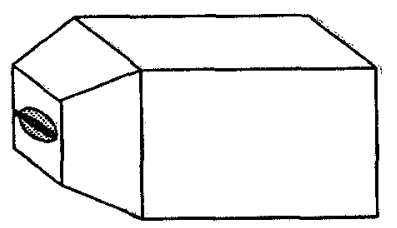

Trimmed block for microtome

Figure $\|$ The preparation of TEM samples using the SEDNB technique. 

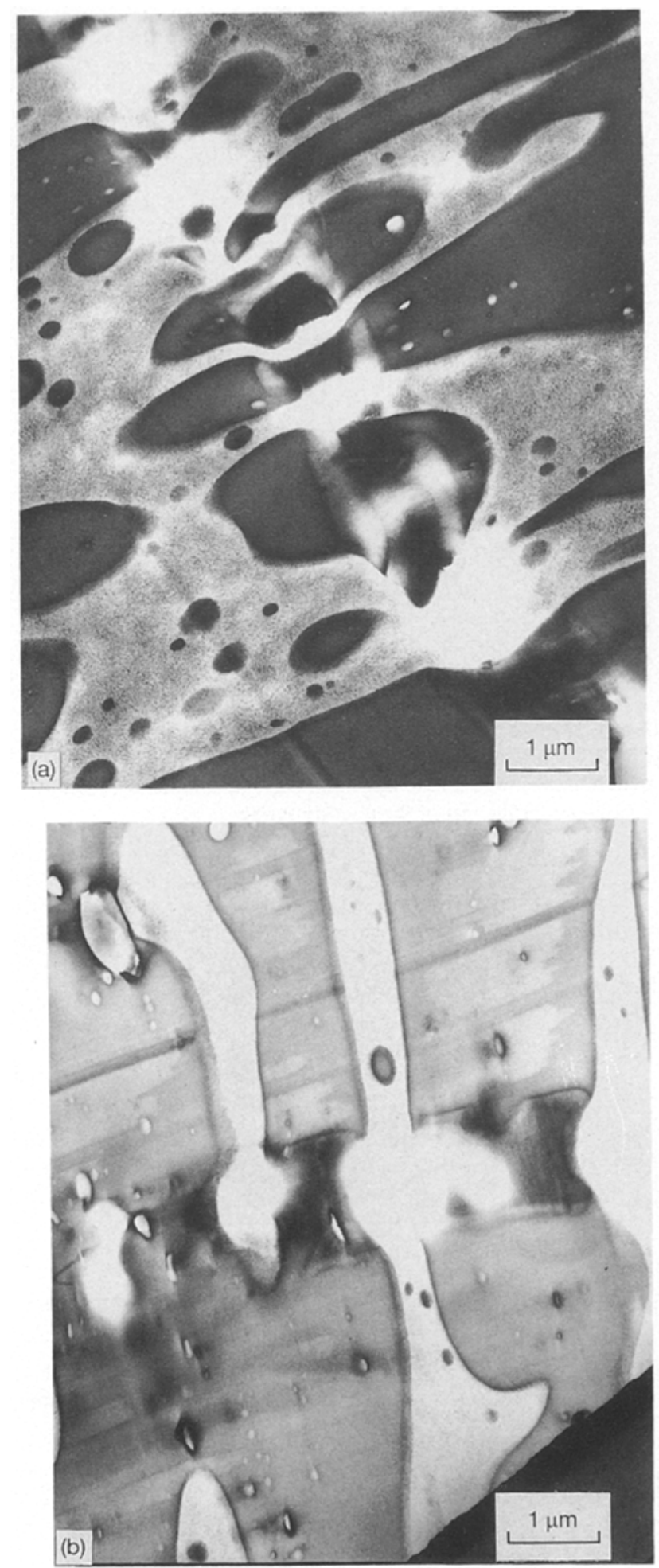

plane-strain constraint is relieved by the voids. Undoubtedly, the plastic-void expansion in this stage also consumes a certain amount of energy. Fourthly, massive plastic deformation occurs in the polymer ligament between neighbouring voids and a tremendous amount of energy is absorbed at this stage. Fifthly, the crack propagates by breaking up the PBT domains but the two surfaces of the crack are still bridged by the highly deformed PC domains. The stress applied at this stage is continuously transferred into the materials by the bridging domains which introduce extra plastic deformation after the crack has passed by.

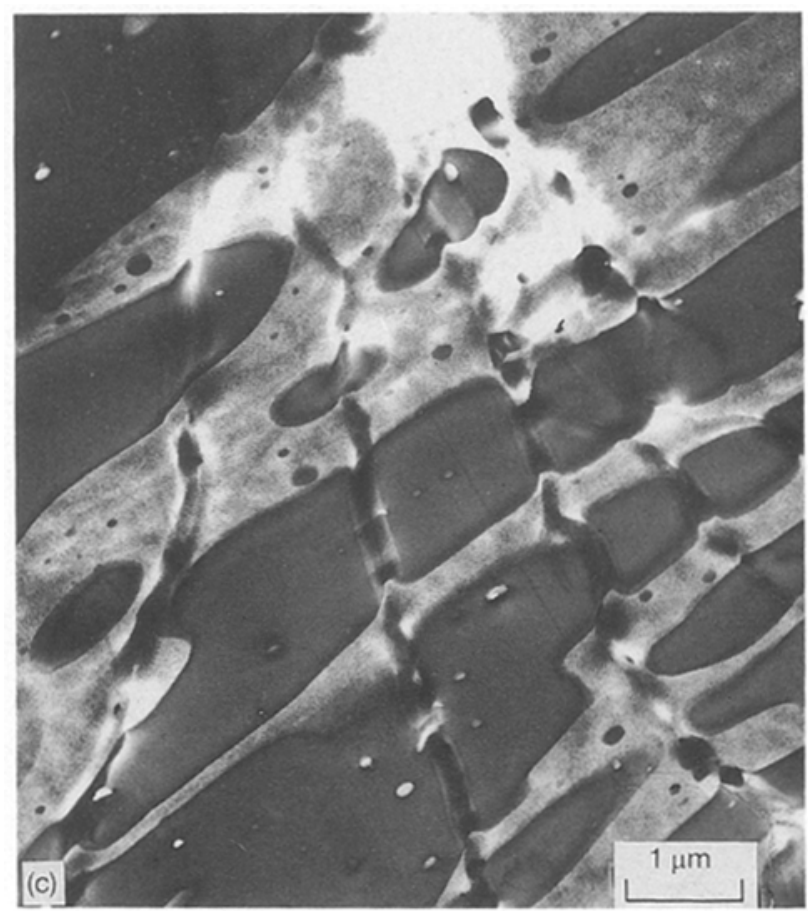

Figure 12 TEM micrographs taken from the PBT/PC $(40 / 60)$ and (50/50) blends. The crack-bridging effect is clearly seen. (a) and (b) PC domains were stretched and yielded. (c) The growing crazes in front of a propagating crack were stabilized by the PC domains.
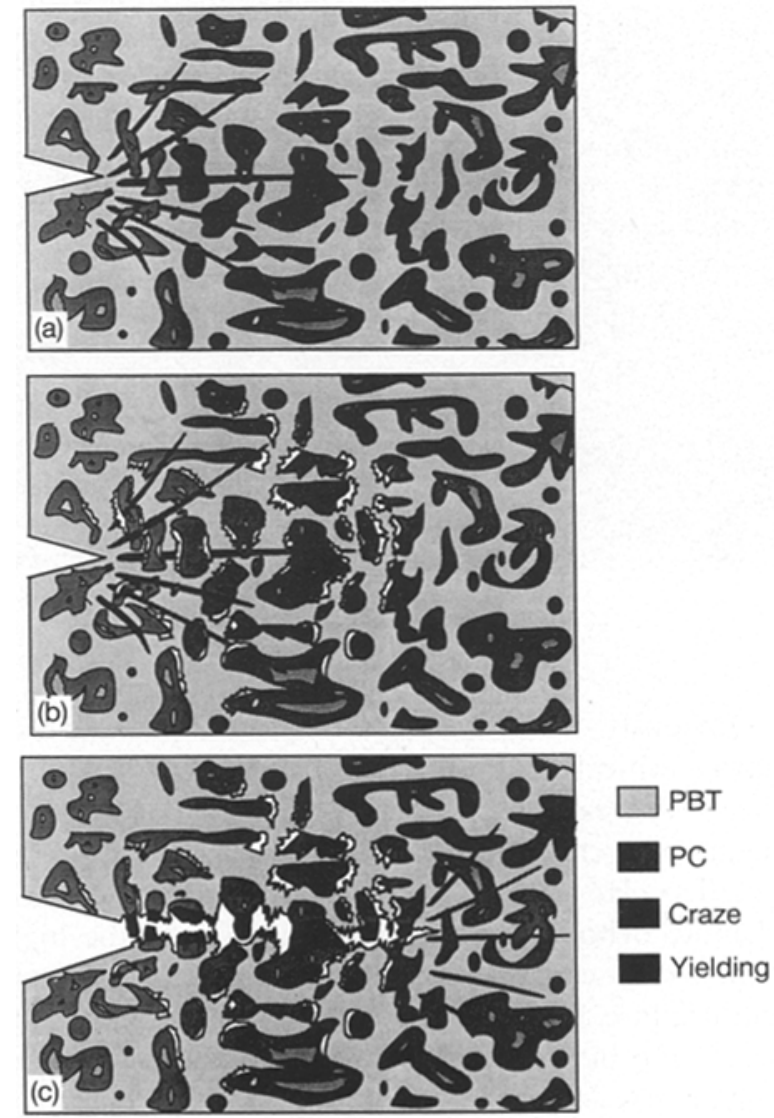

Figure 13 A schematic of the toughening mechanisms observed during the fracture of toughened PBT/PC blends. (a) The crazes initiated by triaxial stress are stabilized by PC domains and the triaxial stress rises to a higher level; (b) with increases in the triaxial stress, debonding-cavitatioin occurs at the PBT/PC phase boundary, introducing localized voids; and (c) extensive shear yielding occurs in the polymer ligaments between the neighbouring voids, and the crack surfaces are bridged by the PC domains. 

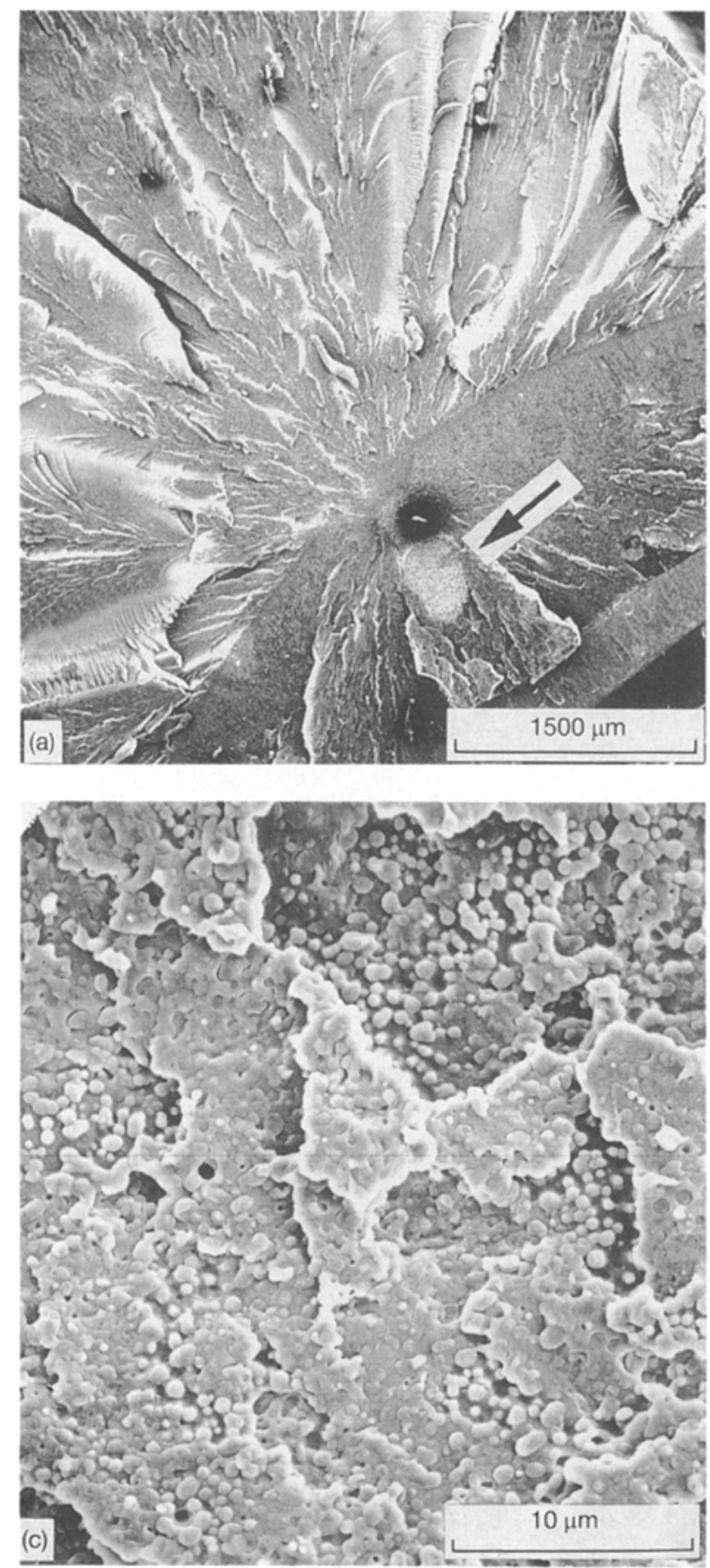

Obviously, the interfacial strength of PBT and PC plays a critical role in the toughening of the $\mathrm{PBT} / \mathrm{PC}$ blends. A weak interfacial adhesion cannot stabilize the growing crazes and consequently it has no bridging effect. However, a very strong interface will not promote debonding-cavitation, as a result, the high plane-strain constraint will inevitably bring about brittle failure. But what level of interfacial strength will benefit the blends most still remains unknown. The domain size is another important factor which may substantially affect the toughness of the blends. For example, very small domain particles are unable to terminate the growing craze, nor can they bridge the crack surfaces. Finally, the mechanical properties of the bridging domains, such as the modulus, the yield stress and the ultimate elongation, are also very important. The bridging domains should probably have

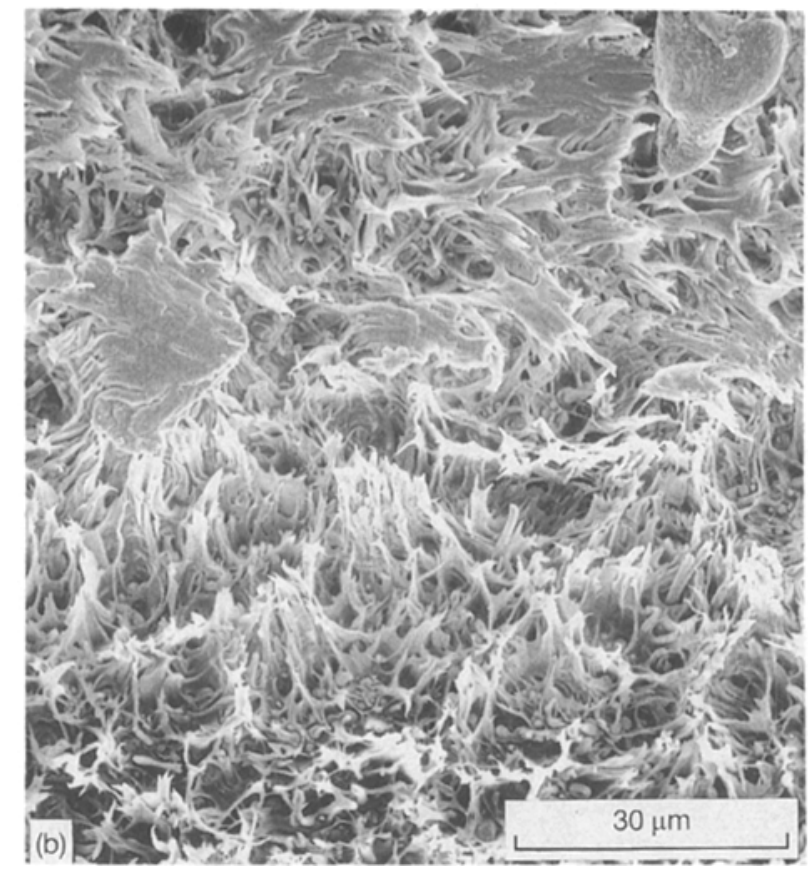

Figure 14 (a) A SEM micrograph of an entire fracture surface of a $\mathrm{PBT} / \mathrm{PC}(60 / 40)$ blend, (b) limited plastic deformation in the crackinitiation area, and (c) the large number of $\mathrm{PC}$ nodules caused by debonding on the rest of the fracture surface.
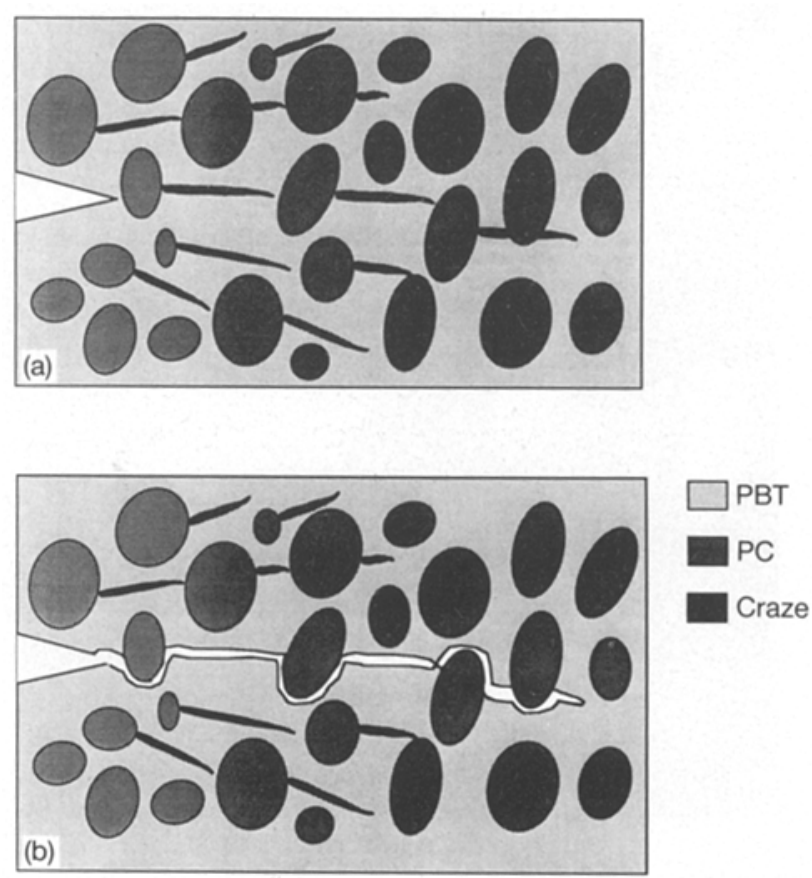

Figure 15 A schematic of the fracture processes observed with PBT/PC blends with PBT as a major component: (a) the crazes initiating at the crack-tip region by triaxial stress, and (b) the development of crazes into cracks and their passage through the poor interface of PBT/PC which eventually introduces a fast unstable fracture

a slightly higher yield stress than the surrounding matrix and as large an ultimate elongation as possible. The answers to all these problems are still not clear. It deserves much more research effort; for, once these questions are clearly answered, a new class of polymer blends with high toughnesses can be created without 

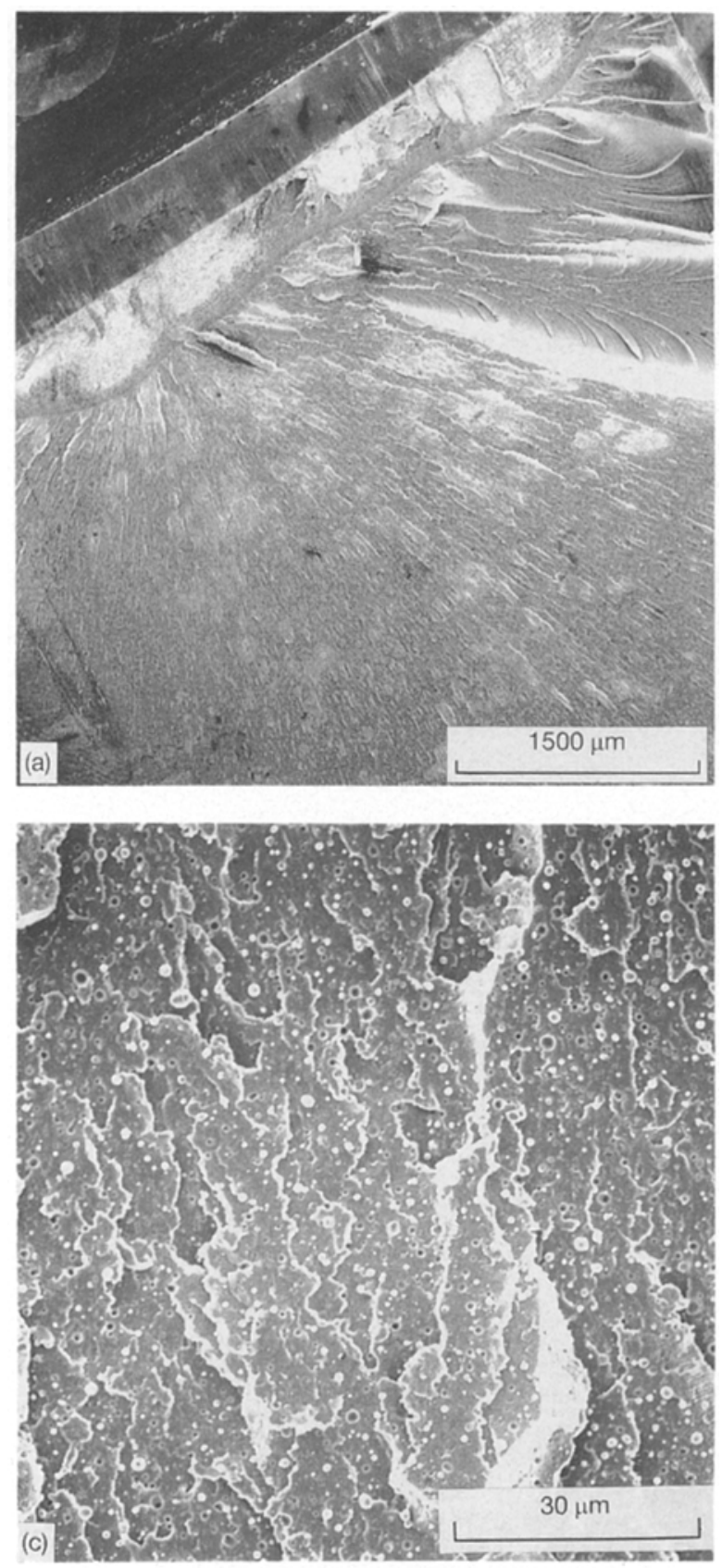

sacrificing other important thermomechanical properties, such as the modulus, the yield stress, the heat tolerance and the chemical resistance.

\section{Conclusion}

1. A series of PBT/PC blends without rubbery particles was made using a twin-screw extruder. The morphology of the blends was studied by TEM and SEM. It was found that the PBT/PC (50/50) blend possessed a bicontinuous structure. The other blends had either a dispersed PBT phase when PBT was the minor component or a dispersed PC phase when PC was the minor component. A relatively strong interface was observed in the blends with $20 \%, 40 \%$ and $50 \%$ PBT. Although no direct evidence was obtained in the present study, based on information from the literature, this strong interface is believed to be caused by the block copolymer generated via PBT/PC transesterification during blending. Poor interfacial adhe-

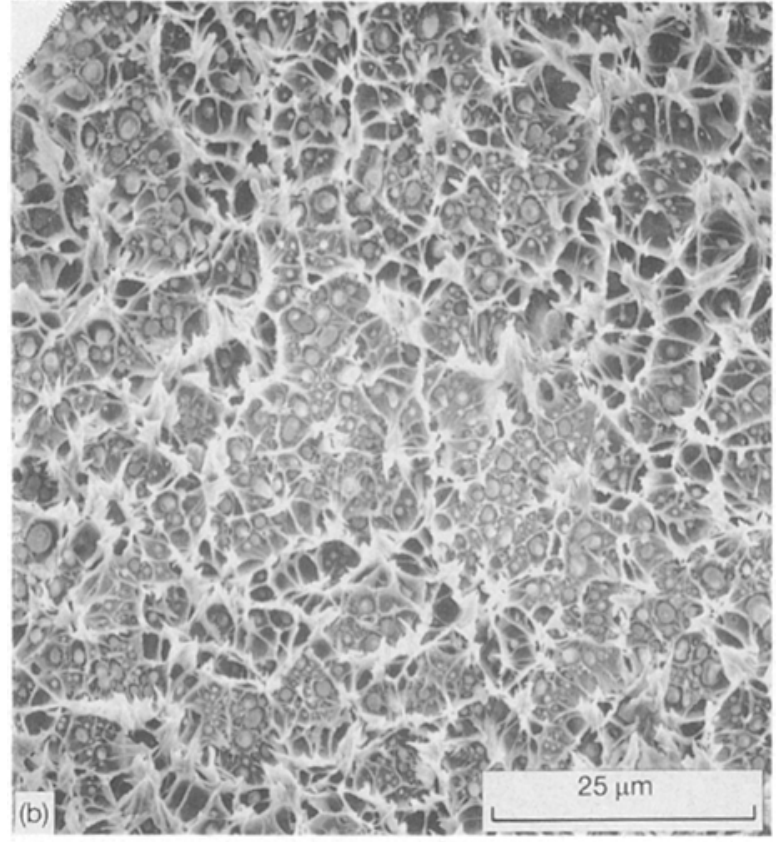

Figure 16 (a) SEM photographs taken from the fracture surface of a PBT/PC $(80 / 20)$ blend. (b) The limited plastic deformation which can be seen in the crack-initiation area. Holes and PC nodules caused by debonding can be clearly seen on the fracture surface. (c) The fast unstable fracture area.

sion is found in the blends with $60 \%$ and $80 \%$ PBT. The reason for this poor interface is tentatively attributed to the low melt viscosity in the blending of these two polymers.

2. The results of the mechanical tests show that the sharp-notch fracture toughness of PC can be significantly increased by mixing with up to $50 \%$ PBT without reducing its modulus and yield stress.

3. The toughening mechanisms involved in the fracture processes of the PBT/PC blends were studied using both SEM and TEM together with SEDNB specimens. It was found that in the toughened blends the crazes in front of a propagating crack were stabilized by the PC domains. Debonding-cavitation occurred at the PBT/PC interface, which relieved the plane-strain constraint and promoted massive shear deformation in the polymer ligament between the neighbouring voids. This plastic deformation absorbed a large amount of energy. Crack bridging by the PC domains was clearly verified by the TEM study. It is proposed that the PC domains not only stabilize the growing crazes but that they also bridged the crack surfaces, thus enhancing the fracture toughness.

4. The poor crack resistance found in the blends rich in PBT was caused by the poor interfacial adhesion between PBT and PC. In these polymer blends, the growing crazes easily developed into cracks, which subsequently passed through the weak interface of $\mathrm{PBT} / \mathrm{PC}$ and finally produced a fast unstable fracture giving a low fracture toughness.

\section{Acknowledgement}

One of us (J. Wu) was financially supported by a Sydney University Postgraduate Research Award and 
a part-time junior research fellowship founded by the Australia Research Council (ARC). The experimental work, which was performed at the University of Michigan, was part of a program supported by E. I. du Pont de Nemours and Company. The donation of materials by G. E. Plastics and the Dow Chemical Company is greatly appreciated.

\section{References}

1. J. G. WILLIAMS, Fracture mechanics of polymers, (Ellis Horwood, Chichester, 1984).

2. J. S. WU, Y. -W. MAI and B. COTTERELL, J. Mater. Sci., 28 (1993) 3373.

3. J. S. W U and Y.-W. MA I, J. Mater. Sci., (1993) 28 (1993) 6167.

4. H.-J. SUE, PhD thesis, University of Michigan (1988).
5. D. S. PARKER, H.-J. SUE, J. HUANG and A. F. YEE Polymer 31 (1990) 2267.

6. A. W. BIRLEY and X. Y. CHEN, Brit. Polym. J. 16 (1984) 77.

7. J. DEVAUX, P. GODDARD and J. P. MERCIER, J. Polym. Sci., Polym. Phys. Edn., 20 (1982) 1881.

8. Idem, ibid. 20 (1982) 1895.

9. B. D. HANRAHAN, S. R. ANGELI and J. RINT, J. Polym. Bull. 14 (1985) 399.

10. J. DEVAUX, P. GODDARD and J. P. MERCIER, Polym. Engng Sci., 22 (1982) 229.

11. J. P. MERCIER, J. DEVAUX and P. GODDARD, British Pat 1569296 (1977).

12. D. C. WAHRMUND, D. R. PAUL and J. W. BARLOW, 22 J. Appl. Polym. Sci., (1978) 2155.

Received 17 May

and accepted 17 December 1993 